\title{
Analytical and Numerical Temperature Prediction in Direct Metal Deposition of Ti6Al4V
}

\author{
Benoit de La Batut ${ }^{1}$, Omar Fergani ${ }^{2, *}$, Vegard Brotan ${ }^{3}$, Markus Bambach ${ }^{4}$ and \\ Mohamed El Mansouri 5 \\ 1 Ecole Centrale de Lyon, 69134 Ecully Cedex, France; benoit.de-la-borie-de-la-batut@ecl14.ec-lyon.fr \\ 2 Department of Mechanical and Industrial Engineering, NTNU, Richard Birkeland's vei 2B, \\ 7010 Trondheim, Norway \\ 3 SINTEF Manufacturing, 7010 Trondheim, Norway; vegard.brotan@ntnu.no \\ 4 Chair for Mechanical Design and Manufacturing, BTU Cottbus-Senftenberg, 03046 Cottbus, Germany; \\ bambach@b-tu.de \\ 5 ENSAM, MSMP Laboratory (EA-7350), 2 cours des Arts et Métiers, 13617 Aix-en-Provence, France; \\ mohamed.elmansori@ensam.eu \\ * Correspondence: o.fergani@ntnu.no; Tel.: +49-162-1611779
}

Received: 18 May 2017; Accepted: 18 June 2017; Published: 10 July 2017

\begin{abstract}
Direct Metal Deposition (DMD) is an additive manufacturing (AM) process capable of producing large components using a layer by layer deposition of molten powder. DMD is increasingly investigated due to its higher deposition rate and the possibility to produce large structural components specifically for the aerospace industry. During fabrication, a complex thermal history is experienced in different regions of the workpiece, depending on the process parameters and part geometry. The thermal history induces residual stress accumulation in the buildup, which is the main cause of distortions. In order to control the process and enhance the product quality, the understanding of the workpiece temperature is substantial. In this study, two methods to predict temperature evolution during the DMD process are introduced based on analytical and finite element methods. The objective is to compare these methods to experimental results and to provide more insights about their capabilities to predict accurately the temperature gradient, the cooling rate, and the melt pool geometry. A comparison of the computational time is also provided. Based on the results of the investigation, It appears that the analytical method provides an effective and accurate method to understand the influence of the process on the workpiece temperature.
\end{abstract}

Keywords: additive manufacturing; direct metal deposition; temperature; prediction; analytical; finite element; melt pool

\section{Introduction}

Direct metal deposition (DMD) also called direct energy deposition (DED) is an advanced additive manufacturing (AM) technology used to repair and rebuild worn or damaged components, to manufacture new components, and to apply wear- and corrosion-resistant coatings [1]. Recently, Liu et al. [2] demonstrated that the technology is capable of manufacturing large aerospace structural components, achieving substantial economical advantages compared to traditional manufacturing processes.

Due to the complexity of the DMD process as described in the work of Bikas et al. [3], it is cumbersome and time-consuming to develop a systematic experimental understanding of the effect of process parameters on part properties. The numerical simulation appears to be the only effective way to achieve an understanding of the DMD process. Furthermore, the in situ technologies offered today are not yet capable of proposing a robust process monitoring for laser processing Thompson et al. [4]. 
Accordingly, modeling offers a suitable way to define process conditions for the DMD process and to monitor and control it online. The final objective being the control of the thermal stresses induced by the non-uniform heating. In fact, the process large thermal gradient $\left(10^{6} \mathrm{~K} / \mathrm{m}\right)$ and cooling rate $\left(10^{5} \mathrm{~K} / \mathrm{s}\right)$ often generate complex microstructure features as demonstrated by the work of Rai et al. [5].

The importance of the temperature prediction is not limited to DMD process control. Several other researchers used the temperature in AM processes to predict microstructure evolution and residual stresses similarly to the work of Kelly [6]. Fergani et al. [7] introduced the first analytical model to predict analytically the residual stresses in AM processes. They based the analysis on the analytical prediction of temperature using a moving heat source. The temperature prediction can be based either on analytical or numerical methods $[8,9]$.

Despite the quite recent industrial expansion of this process, several researchers have investigated different aspects related to the modeling of the temperature in DMD processes using both analytical and FE-based methods. Hoadley and Rappaz [10] introduced a 2D quasi stationary model to predict the temperature in the laser cladding process. They focused in the study on the influence of the laser speed and power on the layer thickness. Picasso and Hoadley [11] introduced a model including the hydrodynamics of the process including the influence of the surface tension and the Marangoni effect. Toyserkani et al. [12] developed a 3D model, the model solved the heat problem using a coupled multi-physics system. They predicted the melt pool shape based on the thermal analysis. In $[4,13]$, the authors provided a comprehensive physics-based overview of the phenomena happening during DMD and proposed a method to optimize and control the process and obtain the desired mechanical properties. Recently, Cao and Ayalew [14] developed a control-oriented multiple input multiple output modeling of laser-aided powder deposition processes. The objective of their work is to control the height and the temperature of a layer. The study demonstrated the important role of temperature modeling to control the quality of the final component.

Several studies have used the finite elements method FEM to investigate the DMD process [15-17] using classical solvers for thermo-mechanical problems. Most of the studies neglected the hydrodynamics aspect of the molten metal by implementing a well-designed volume heat flux as shown by the work of $\mathrm{Li}$ et al. [18]. Other than predicting the temperature and the thermal stresses, the FE-based approach serves also to predict the melt pool geometry as proposed by Peyre et al. [15] using a 3D FE model. Several other researchers in [19-21] have proposed a complete multi-physics models to predict both the thermo-mechanical processing experienced by the material as well as the complex laser-materials interaction leading to fluid flow in the melt pool, surface tension and thermocapillary forces. Morville et al. [22] and Morville [23] presented simulations of a single layer and multilayer cladding with fluid flow and heat transfer to demonstrate the capabilities of FEM method to give a good comprehension of the phenomena responsible for a deleterious surface finish. The authors used COMSOL to implement a moving mesh that captures the dynamic shape of the molten zone. They also compared the geometry simulated to experimental data obtained with a high speed camera. One of the main drawbacks of FE-based methods is the large computational cost, although improvement are investigated to speed up such models as demonstrated by Klindworth et al. [24].

One of the main challenges related to the DMD process is the deposition method. Theoretical and experimental analysis to compare coaxial cladding from the traditional side-cladding set-up is performed by de Oliveira et al. [25]. Unfortunately, most of the studies in the literature used empirical models to capture the deposition method. Morville et al. [22] considered a priori geometries as in Paul et al. [17]. Recently, an increasing number of studies tend to implement a coupled heat transfer and phase transition during the additive manufacturing processes to have a better understanding of the physics near the melt pool and thus a more accurate temperature prediction. In a study of the selective laser melting process, Li et al. [26] developed a model capturing the solid, liquid and vapor phases as well as the induced volume shrinkage to quantify the influence of vaporization during the process. 
To the best knowledge of the authors, no previous studies presented a comparison of these two methods in terms of computational time, accuracy and ease of implementation. It is known that the FE-based method is slow, but it can be accelerated by Model Order Reduction (MOR) techniques, yielding, however, a large coding effort. Analytical models are fast but may give limited accuracy. The main motivation of this study is to provide the basis of a comparison between the analytical and the numerical methods from both accuracy and the cost of computations.

This study aims to develop and compare two methods of predicting the temperature during the DMD process. A 2D model is developed using both analytical and numerical approaches. After the review of the literature and the problem formulation, Section 2 presents the mathematical and practical details of the numerical and the analytical temperature models. Section 3 presents the details of the material properties and the experimental data used as a baseline for the proposed comparison. In Section 4, the results are presented and discussed using a comparative study. The sensitivity of the temperature to the main process parameter variation is investigated using the analytical model. Section 5 concludes this study.

\section{Mathematical Modeling of the DMD Process}

\subsection{Numerical Modeling}

The aim of this section is to introduce the numerical models used for the calculation of the temperature distribution inside the material when a laser is moved along its surface while depositing the molten metal. For this purpose, COMSOL multi-physics software is used to run an FEA on a 2D geometry (Figure 1). In this approach, the fluid flow is not studied, even though the phase change and its consequences on the material characteristics are implemented, the liquid is supposed to remain still in the melt pool. The approach is also limited to the workpiece, the interaction between the surrendering gas and the material is neglected. The simulation aims at understanding the temperature after solidification. After this bright moment, convection in the melt pool may be neglected.

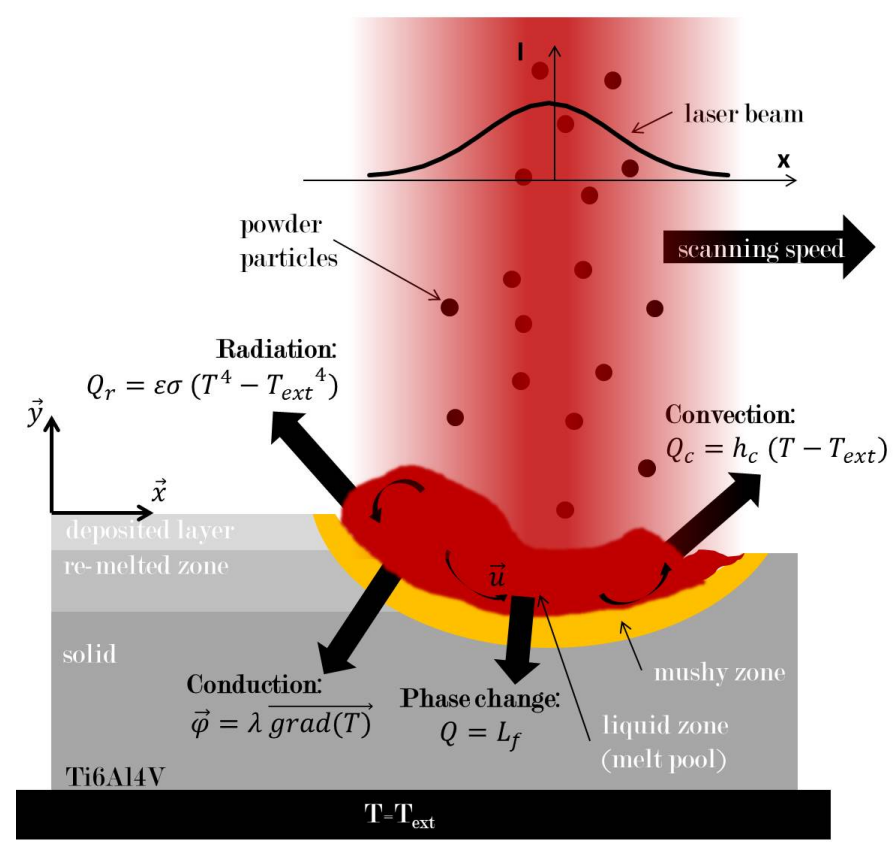

Figure 1. Heat transfer and configuration of the DMD process.

The temperature inside the material is ruled by the energy conservation equation (Equation (1)), where $T$ is the temperature and $\lambda$ the thermal conductivity. The right-hand side of the equation 
accounts for the variation of internal energy. The first term on the left-hand side represents the heat conduction:

$$
\rho c_{p}^{*}\left(\frac{\partial T}{\partial t}+\vec{V} \cdot \vec{\nabla} T\right)=\vec{\nabla} \cdot(\lambda \vec{\nabla} T)+Q_{v} .
$$

$c_{p}^{*}$ is the modified heat capacity, which takes into account the phase change in the material: $c_{p}^{*}=c_{p}(T)+L_{f} \frac{\partial f_{l}}{\partial t}$, where $c_{p}(T)$ is the heat capacity, $L_{f}$ the latent heat of fusion and $f_{L}$ the latent heat of fusion, supposed to be a linear function of $T$ in the mushy zone $\left(T_{S}\right.$ and $T_{L}$ are the solidus and liquidus temperatures.):

$$
f_{L}=\left\{\begin{array}{cc}
0 & T<T_{S} \\
\frac{T-T_{S}}{T_{L}-T_{S}} & T_{S} \leq T \leq T_{L}, \\
1 & T_{L}<T .
\end{array}\right.
$$

$Q_{v}$ in Equation (1) is a negative heat flux used to model the heat loss in the $z$-direction at the two large walls of the material. It accounts for both convection and radiation as it was introduced in the work of Morville [22]. This term must not be forgotten as the geometry simulated is rather thin $\left(w_{0}=2 \mathrm{~mm}\right) . T_{0}$ is the outside temperature, $h_{c}$ is the convection coefficient, $\sigma_{b}$ the Stefan-Boltzmann constant and $\epsilon$ the emissivity:

$$
Q_{v}=-2 \frac{h_{c}\left(T-T_{0}\right)+\epsilon \sigma_{B}\left(T^{4}-T_{0}^{4}\right)}{w_{0}} .
$$

In order to solve the set of equations, simplifications are required. It is supposed that the geometry of the workpiece remains constant (no layer addition). In particular, the top boundary will not be affected by the fluid flow and no evaporation of the material throughout this surface is accounted for. Finally, the material is set to be homogeneous and isotropic.

The model is completed by the following boundary conditions, constant over time. On the bottom surface, the temperature is supposed to remain constant and at the temperature of the substrate. This is acceptable as long as the depth of the workpiece is substantial compared to the size of the melt pool. On the lateral faces of the material, heat loss is modeled by convection and radiation:

$$
\lambda \vec{\nabla} T \cdot \vec{n}=-h_{\mathcal{c}}\left(T-T_{0}\right)-\epsilon \sigma_{B}\left(T^{4}-T_{0}^{4}\right) .
$$

On the top surface, in addition to convection and radiation, a heat flux is added to model the laser:

$$
\lambda \vec{\nabla} T \cdot \vec{n}=I-h_{\mathcal{c}}\left(T-T_{0}\right)-\epsilon \sigma_{B}\left(T^{4}-T_{0}^{4}\right),
$$

where $I$ is of Gaussian distribution, moving at the speed $V$ along the $x$-axis:

$$
I=k \frac{P}{\pi r^{2}} \cdot e^{\left(\frac{x-V \cdot t}{r}\right)^{2}} .
$$

$k$ is a numerical parameter used to fit the experimental data. It accounts for the absorptivity of the material, the heat lost to the metal powder before it falls into the melt pool and the angle of the surface with the laser beam. It corresponds to $A$ and $\alpha_{a}$ in Equation (11). $P$ is the laser power and $r$ the laser sport radius.

At the beginning of the simulation, the temperature in the material is set to the ambient temperature $T_{0}$.

The geometry simulated is divided into two constant meshes. The first one is a rectangular of $80 \mathrm{~mm} \times 10 \mathrm{~mm}$ composed of 49,658 triangular elements of the average surface of $0.01 \mathrm{~mm}^{2}$. The second one is a rectangular mesh of $80 \mathrm{~mm} \times 20 \mathrm{~mm}$ composed of 11,978 triangles of the average surface of $0.6 \mathrm{~mm}^{2}$. The first mesh is at the surface, and it is finer because variations of temperature are more important. To avoid long computation times, the second coarser mesh is added. It must be 
deep enough to ensure that the bottom border will not interfere with the temperature on the surface. A convergence study has been done to validate the mesh size. The mesh can be seen in Figure 2.

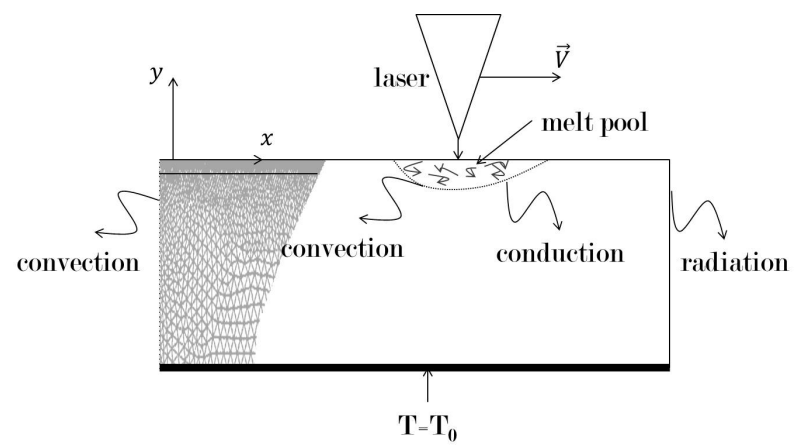

Figure 2. Representation of the mesh and of the numerical model.

\subsection{Analytical Model}

The analytical solution is developed and implemented in Matlab software. The geometry simulated is in two dimensions and is supposed to be semi-infinite. The model solely considers the heat transfer inside the material, without any phase change and without interaction with the surrounding gas. Both convection and conduction inside the material are considered in this section.

Equation (7) is the general convection-diffusion equation for a heat source moving at the constant velocity $V$ in a laminar heat flow [16]. It is valid for an isotropic homogeneous material. $h_{e}$ is the enthalpy:

$$
\frac{\partial(\rho u)}{\partial t}+\frac{\partial\left(\rho h_{e} V\right)}{\partial x}=\nabla \cdot(\lambda \nabla T)
$$

The steady state approximation leads to the elimination of the first term of Equation (7). Furthermore, it can be simplified given that $d u=d h_{e}=c_{p} d T$ :

$$
\rho c_{p} V \frac{\partial T}{\partial x}=\nabla \cdot(\lambda(T) \nabla T) .
$$

The left-hand side of Equation (8) represents the convection in the body; the first part of the right-hand side is the conduction term. Under these assumptions, no variation of internal energy is accounted for in Equation (8).

Carslaw and Jaeger studied such an equation [27] in a semi-infinite body. The solution of the heat diffusion for a point source $q(x)$ at a time $t \rightarrow \infty$ in two dimensions is given in Equation (9). $D$ is the thermal diffusivity of the material $\left(D=\frac{\lambda}{\rho c_{p}}\right)$. In this solution, the material parameters $(D)$ are supposed to remain constant with the temperature:

$$
T=T_{0}+\frac{q(x)}{4 \pi \lambda \sqrt{x^{2}+y^{2}}} e^{\frac{-V\left(\sqrt{x^{2}+y^{2}}+x\right)}{2 D} .}
$$

According to this equation, the temperature at the heat point is infinite. To have a finite solution on the surface, it has to be assumed that the heat point is at an infinitely small distance from the surface.

From this starting point, the equation may be solved for an arbitrarily shaped source by integrating solution (9) over the laser spot domain $\left(\left[x_{s}, x_{e}\right],\left[y_{s}, y_{e}\right]\right)$ as follows [28]:

$$
T(x, y)=T_{0}+\frac{1}{2 \pi \lambda} \cdot \int_{y_{s}}^{y_{e}} \int_{x_{s}}^{x_{e}} \frac{q(u) e^{\frac{-V\left(\sqrt{(x-u)^{2}+y^{2}}-(x-u)\right.}{2 D}}}{\sqrt{(x-u)^{2}+y^{2}}} d u d v .
$$

In this study, the penetration of the laser inside the workpiece is neglected $\left(y_{s}=y_{e}=0\right)$. In order to solve Equation (10), the material properties $\left(\rho, c_{p}, \lambda\right.$, ) are assumed constant with respect to the 
temperature. The material is isotropic and homogeneous. As a consequence of modeling a semi infinite body, the bottom and side boundaries are not added to the model. Furthermore, the equation is solved without heat heat loss on the surface by radiation or convection.

Equation (10) is solved in Matlab under the previous assumptions with a heat flux of Gaussian shape as proposed in $[29,30]$ :

$$
q(x)=\frac{A P \alpha_{a}}{\pi r^{2}} e^{-B\left(\frac{x}{r}\right)^{2}}
$$

where $A$ and $\alpha_{a}$ are the laser absorption coefficient and the workpiece absorptance $\left(A \cdot \alpha_{a}=0.2\right) . B$ is the shape factor of the Gaussian distributed heat flux $(B=2)$ and $r$ is the laser spot radius. Contrary to Equation (6), there is no time variable in this equation, as the equation is solved in the steady state equation. The scanning speed $V$ is only found in Equation (10).

Table 1 gives a summary of the main differences between the two models.

Table 1. Model comparison.

\begin{tabular}{ccc}
\hline & Numerical Model & Analytical Model \\
\hline Material properties & temperature dependent & constant \\
\hline & fixed geometry & fixed geometry \\
\cline { 2 - 3 } Assumption & 2D finite geometry & $\begin{array}{c}\text { 2D semi infinite geometry } \\
\text { no latent heat of fusion } \\
\text { steady state assumption }\end{array}$ \\
\hline Boundary conditions & radiation, convection and laser input & only the laser input \\
\hline
\end{tabular}

\section{Material and Experiment}

The proposed models in this work are implemented and compared to experimental data performed on the DMD process using Ti6Al4V. The DMD machine used is an OPTOMEC LENS $850 \mathrm{R}$ (Optomec Inc., Albuquerque, NM, USA). The dimensions of the samples are $2 \mathrm{~mm}$ in width, $70 \mathrm{~mm}$ in depth and $80 \mathrm{~mm}$ in length. The chemical composition of the alloys powder can be found in Table 2. The temperature on the workpiece surface is measured using a thermocouple of type $\mathrm{K}$ (chromel/alumel), in contrast to the thermal camera, thermocouples are not sensitive to the variation of the emissivity of the material induced by the phase change. In order to control the experimental setup, the authors used a thermal-camera and a high speed camera to provide comparison bases for the temperature and the melt-pool size. The DMD uses a Yb-YAG laser with a wavelength of $1030 \mathrm{~nm}$. The full experimental setup details can be found in the work of Pouzet [31].

Table 2. Chemical composition of Ti6Al4V (\%).

\begin{tabular}{cccccccc}
\hline Ti & Al & V & Fe & O & N & C & H \\
\hline & 6.2 & 4.2 & 0.18 & 0.1 & 0.01 & 0.02 & 0.001 \\
\hline
\end{tabular}

Two parameters are studied: the scanning speed $V(0.2 \mathrm{~m} / \mathrm{min}$ or $0.4 \mathrm{~m} / \mathrm{min})$ and the laser power $P(400 \mathrm{~W}$ or $600 \mathrm{~W})$. The initial temperature of each experiment depends on its final temperature as the process is multi-layered (Figure 3 ). Both $\mathrm{V}$ and $\mathrm{P}$ are parameters in the analytical and numerical simulations that can be set as arbitrary. 


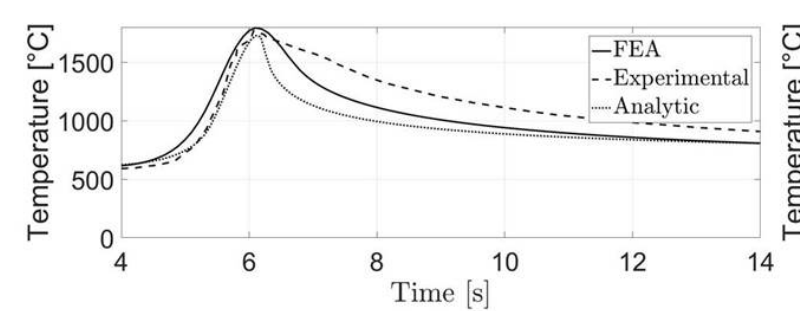

(a)

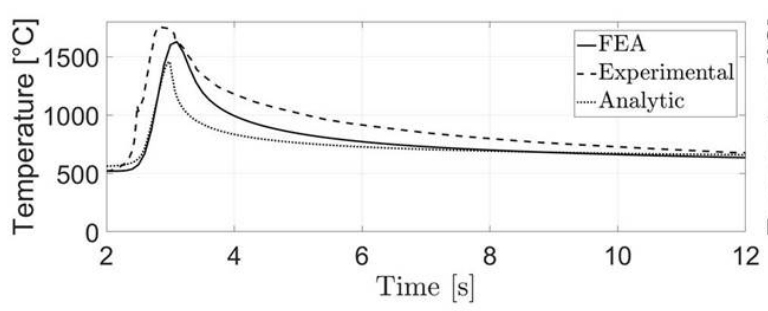

(c)

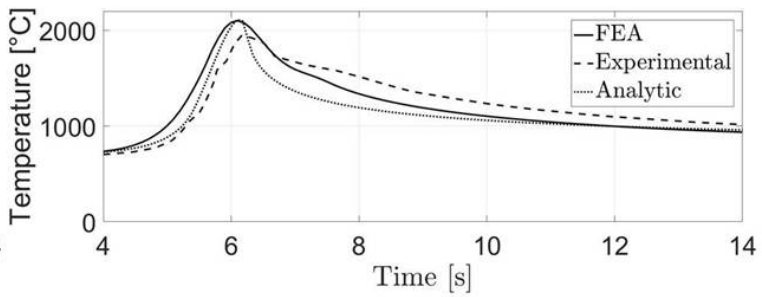

(b)

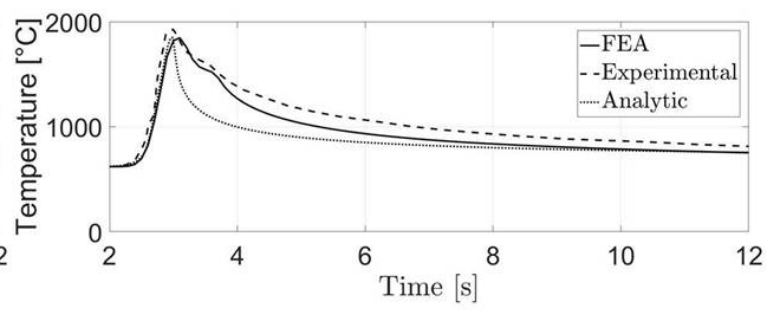

(d)

Figure 3. Comparison of the surface temperature. (a) $P=400 \mathrm{~W}, V=0.2 \mathrm{~m} / \mathrm{min}$; (b) $P=600 \mathrm{~W}$, $V=0.2 \mathrm{~m} / \mathrm{min}$; (c) $P=400 \mathrm{~W}, V=0.4 \mathrm{~m} / \mathrm{min}$; (d) $P=600 \mathrm{~W}, V=0.4 \mathrm{~m} / \mathrm{min}$.

Table 3 gives the values of the process parameters and the material characteristics for Ti6Al4V.

Table 3. Table of the process and material parameters.

\begin{tabular}{|c|c|c|c|}
\hline Symbol & Value & Unit & Name \\
\hline $\mathrm{T}$ & & K & temperature field \\
\hline$\rho$ & 4400 [32] & $\mathrm{kg} / \mathrm{m}^{3}$ & density \\
\hline$c_{p}$ & {$[500-800][32]$} & $\mathrm{J} /(\mathrm{kg} \cdot \mathrm{K})$ & heat capacity \\
\hline$\lambda$ & {$[5-35][32]$} & $\mathrm{W} /(\mathrm{m} \cdot \mathrm{K})$ & thermal conductivity \\
\hline$f_{L}$ & [0-1] & 1 & liquid fraction \\
\hline$T_{L}$ & 1823 & K & liquidus temperature \\
\hline$T_{S}$ & 1773 & K & solidus temperature \\
\hline$T_{0}$ & 25 & ${ }^{\circ} \mathrm{C}$ & ambient temperature \\
\hline$L_{f}$ & $2 \cdot 10^{5}$ & $\mathrm{~J} / \mathrm{kg}$ & latent heat \\
\hline$\sigma_{B}$ & $5.67 \cdot 10^{-8}$ & $\mathrm{~W} /\left(\mathrm{m}^{2} \cdot \mathrm{K}^{4}\right)$ & Stefan-Boltzmann constant \\
\hline$h_{c}$ & 24 [22] & $W /\left(m^{2} \cdot K\right)$ & convection coefficient \\
\hline$\epsilon$ & 0.9 & 1 & emissivity \\
\hline$P$ & [200-400] & W & laser power \\
\hline$I$ & & $\mathrm{~W} / \mathrm{m}^{2}$ & laser heat flux \\
\hline$r$ & 70 & $\mu \mathrm{m}$ & laser spot radius \\
\hline$V$ & {$[0.2-0.4]$} & $\mathrm{m} / \mathrm{s}$ & scanning speed \\
\hline$A$ & & 1 & laser absorption coefficient \\
\hline$\alpha_{a}$ & & 1 & Absorptance of the workpiece \\
\hline$B$ & 2 & 1 & Gaussian shape factor \\
\hline
\end{tabular}

The parameter $k$ in Equation (6) is set to 0.3; this value appears to be acceptable as the absorption coefficient of the titanium powder is between 0.2 and 0.4 [31]. This value of $k$ was set thanks to a sensitivity analysis with the analytical model to fit experimental and numerical data. For the analytical work, all parameters were set as constant to make the resolution possible, the values of the material parameters were chosen as average in the temperature zone of the experiment: $C_{p}=700 \mathrm{~J} /(\mathrm{kg} \cdot \mathrm{K})$, $\lambda=17 \mathrm{~W} /(\mathrm{m} \cdot \mathrm{K})$ and $\rho=4400 \mathrm{~kg} / \mathrm{m}^{3}$. For the numerical work, the emissivity was also set as a constant and was used as a fitting parameter. A deeper study on its variation could be beneficial. 


\section{Results and Discussion}

The capabilities of both analytical and numerical models are compared to the experimental results. Some of the most important aspects investigated in this section are the temperature gradient and the cooling rate. The iso-temperature lines are compared for similar temperature parameters. Additionally, the steady state temperature is compared.

\subsection{Surface and Subsurface Temperature}

Figure 3 represents the evolution of the surface temperature during the DMD process at the steady state. Different combinations of process parameters are represented, specifically the scanning speed and the laser powder. The results of the analytical, numerical and experimental results are compared. The temperature profile on a point on the surface is presented at the steady state. Peaks on the curves correspond to the moment that the laser is above the studied point. The curves are composed of the heat of the part, until the peak, and then the cooling at a slower rate. Discrepancies are observed in terms of cooling rates and analysed in the next section. On Figure $3 \mathrm{~b}, \mathrm{~d}$, a plateau behavior is observed during the cooling of the workpiece, which is due to the phase transition of the metal from liquid to solid. This is not seen in the analytical work, as the latent heat of the material is not taken into account in the model.

Overall, the temperature on the surface in terms of magnitude is well captured by both analytical and numerical approaches. The numerical model better approached the experimental measurements. This is expected as the model captures multi-physics; specifically, the phase changes happening at the melting point as well as the influence of the temperature on the materials' properties.

Despite the capability to accurately predict the temperature profile on the surface using the numerical modeling, the analytical approach provides the advantage of a short computational time.

Figure 4 compares the temperature at different depths inside the workpiece. Both results from the numerical and analytical work are represented. The hot point in red corresponds to the position of the laser.

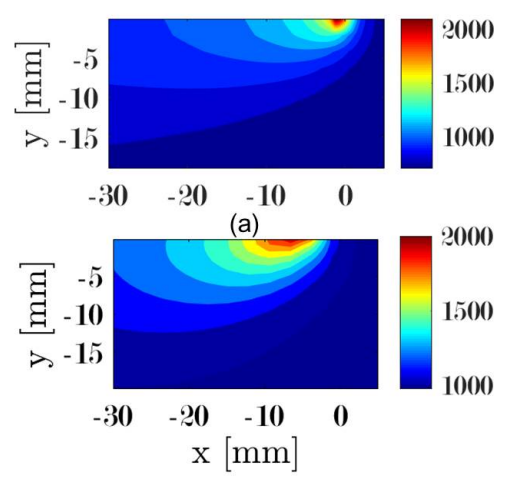

(c)

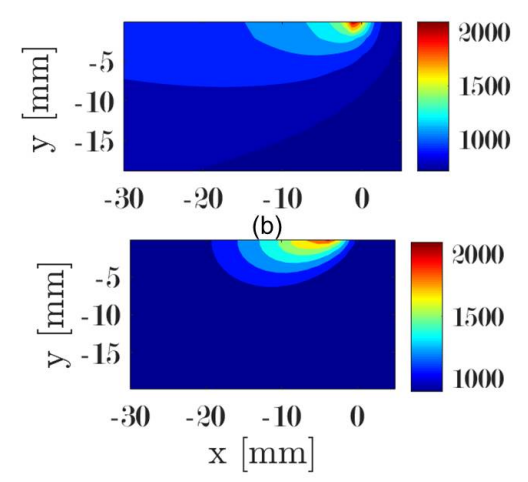

(d)

Figure 4. Comparison of analytical and numerical works $\left({ }^{\circ} \mathrm{C}\right) .($ a) analytical model $(P=600 \mathrm{~W}$, $V=0.2 \mathrm{~m} / \mathrm{min}) ;($ b) analytical work $(P=600 \mathrm{~W}, V=0.4 \mathrm{~m} / \mathrm{min}) ;(\mathrm{c})$ numerical model $(P=600 \mathrm{~W}$, $V=0.2 \mathrm{~m} / \mathrm{min})$; (d) numerical work $(P=600 \mathrm{~W}, V=0.4 \mathrm{~m} / \mathrm{min})$.

It can be seen that the analytical model predicts higher temperatures on the surface compared to the FE-based model. In other words, the heat-affected zone spreads wider in the analytical work. This can be explained by the fact that the proposed analytical model does not capture the heat loss on the surface through convection and radiation. This approximation appears acceptable as long as the temperature is calculated close to the heat source where the radiation is small compared to the heat input of the laser. 
When the melt pool geometries from the analytical and numerical approaches are compared in Figure 5, it appears that the numerical model gives a melt pool $27 \%$ larger. The depth of the two melt pools are comparable; however, the numerical model predicts a wider melt pool in all of the simulated cases.

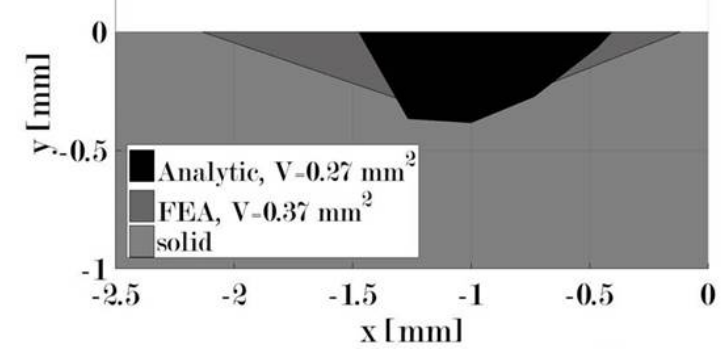

Figure 5. Melt pool geometry.

\subsection{Cooling Rate Comparison}

The influence of the scanning speed on the temperature distribution is studied in this section. The temperature is calculated on the top surface, where it is higher, for the analytical and numerical model, as well as for the experimental data. The maximum cooling rate is equal to $1634 \mathrm{~K} / \mathrm{s}$ for a scanning speed of $0.2 \mathrm{~m} / \mathrm{s}$ and $3297 \mathrm{~K} / \mathrm{s}$ for a scanning speed of $0.4 \mathrm{~m} / \mathrm{s}$. Figure 6 represents the cooling rate of the workpiece on the surface during the surface. The analytical cooling rate can be calculated as the steady state approximation links the time and the $x$-dimension.

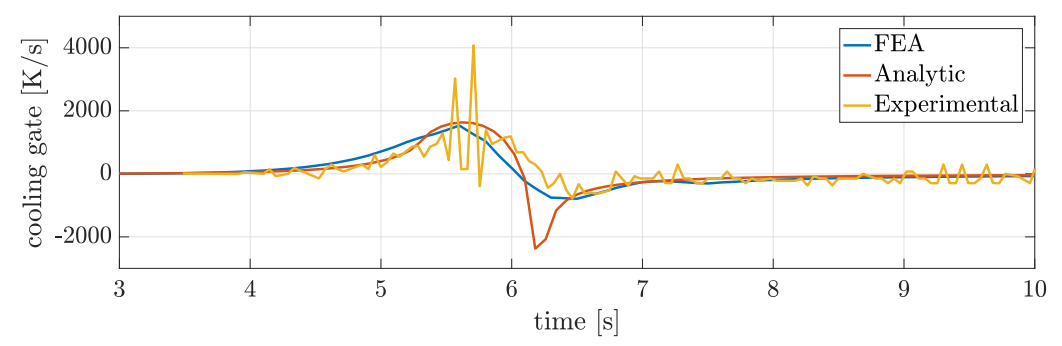

(a)

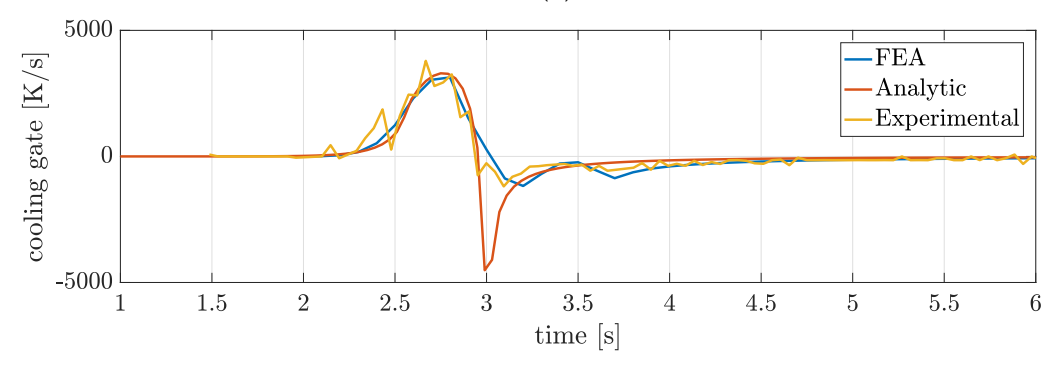

(b)

Figure 6. Comparison of the different cooling rate on the surface (a) $P=600 \mathrm{~W}, V=0.2 \mathrm{~m} / \mathrm{min}$; (b) $P=600 \mathrm{~W}, V=0.4 \mathrm{~m} / \mathrm{min}$.

The curves are divided into two: in the first half, the laser is approaching the studied point, and the cooling rate is positive as the temperature in the material increases. The curves go through a maximum when the laser is on the studied point. In the second half, the laser moves away and the temperature starts to decrease (negative cooling rate). The numerical approach and the experimental data show close results on the two parts of the curves, even if the experiments presented some peaks that are not captured by the models. 
The analytical work gives good results in the first half of the curve, but it overestimates the cooling rate in the second one, as it gives a minimum cooling rate more than twice as big as the others. This result is explained by the constant material parameters that accounts for a much colder material at the moment when the temperature is at its highest. The scanning speed has a strong influence on the maximum cooling rate.

An important limitation of the analytical work presented here is that the characteristics of the material are supposed to remain constant with the temperature. However, studies show that this is not the case [32]. The thermal conductivity $\lambda$ and the heat capacity, particularly, will be greatly affected by the change of temperature. Between $300 \mathrm{~K}$ and $2000 \mathrm{~K}$, their values will, respectively, vary between $5 \mathrm{~W} /(\mathrm{m} \cdot \mathrm{K})$ and $30 \mathrm{~W} /(\mathrm{m} \cdot \mathrm{K})$ and $550 \mathrm{~J} /(\mathrm{kg} \cdot \mathrm{K})$ and $800 \mathrm{~J} /(\mathrm{kg} \cdot \mathrm{K})$. Such variations have consequences for the analytical results and the values chosen for thermal capacity and thermal conductivity must be set wisely.

In order to provide a full picture of the capabilities of the analytical and FE-based model, the computation cost is investigated. The main advantage of such an approach is the speed of the computation: its computation time is a few seconds, whereas the numerical computation takes about 30 min of calculation (CPU: Intel ${ }^{\circledR}$ Xeon ${ }^{\circledR}$ CPU W3680 @ $3.33 \mathrm{GHz}$, six cores). Furthermore, the analytical approach as of now can only model one scan, as it can't have a complex initial temperature field from a previous layer deposition. However, the cooling rate is accurately predicted in its most critical phase, at its peak. This is the most important part of the curve, as it directly linked to the martensite formation in the material. This model allows for a good comprehension of the microstructure evolution.

\subsection{Sensitivity Analysis Based on the Analytical Model}

In order to understand the influence of the process parameters on the temperature, cooling rate, and melt pool, a sensitivity study is designed to investigate both the laser scanning speed and the laser power. In each case, one was fixed and the other was set to different values. The small computational time associated with the analytical modeling approach allows for a better understanding of the influence of the process parameters as discussed previously.

\subsubsection{Influence of the Laser Power}

Figure 7 gives the influence of the laser power on the temperature as predicted by the analytical model. The scanning speed is set to $0.4 \mathrm{~m} / \mathrm{s}$ and the laser power varies between $100 \mathrm{~W}$ and $700 \mathrm{~W}$.

As expected, the increase of the laser powder increases the surface temperature (Figure 7a). The results of the simulation using the analytical model suggest that the maximum temperature and cooling rate vary linearly with the laser power (Figure $7 \mathrm{~b}$ ). The same variation is observed in the cooling rate (Figure $7 \mathrm{c}, \mathrm{d})$.

The analysis of the geometrical shape of the melt pool shows an increasing cross-sectional area with the increase of the laser power (Figure 7e). A power law correlation is established between the laser power and the melt pool area, while a linear type of dependence exists between the laser power and the temperature. 


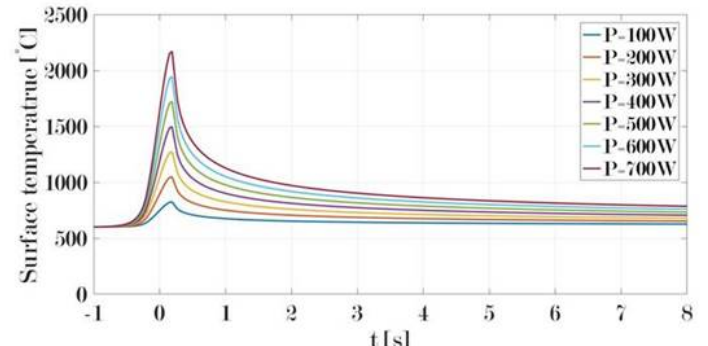

(a)

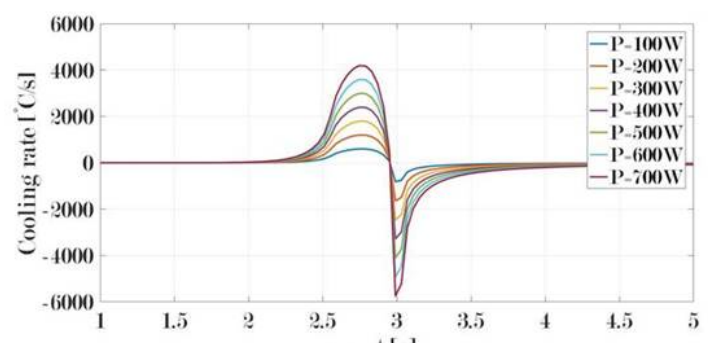

(c)

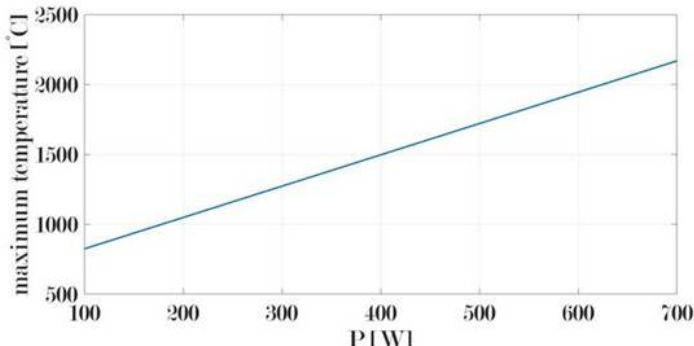

(b)

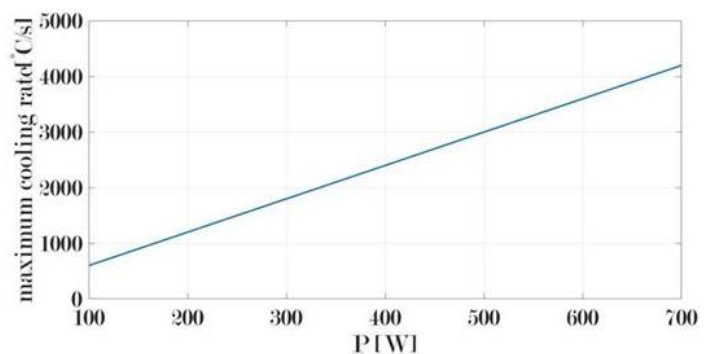

(d)

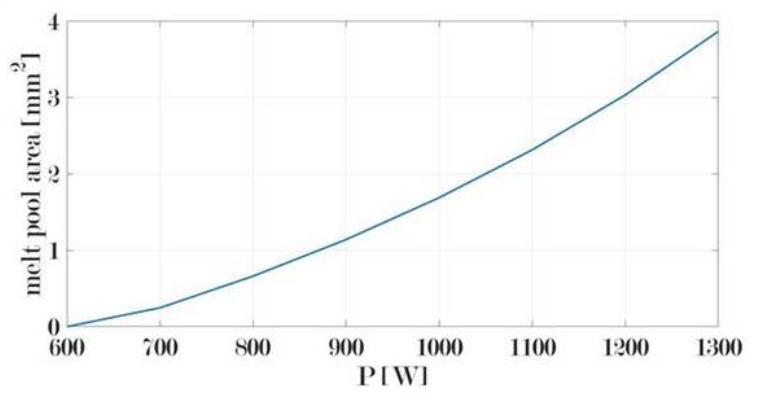

(e)

Figure 7. Simulation of the influence of the laser power on the temperature and on the melt pool $(V=0.4 \mathrm{~m} / \mathrm{s})(\mathbf{a})$ surface temperature for different values of the laser power; (b) evolution of the maximum surface temperature with the laser power; (c) surface cooling rate for different values of the laser power; (d) evolution of the maximum surface cooling rate with the laser power; and (e) evolution of the melt pool cross section with the laser power.

\subsubsection{Influence of the Scanning Speed}

The influence of the scanning speed is studied in Figure 8 with a laser power of $400 \mathrm{~W}$ and a range of scanning speed of $[0.1 \mathrm{~m} / \mathrm{s}-0.6 \mathrm{~m} / \mathrm{s}]$ corresponding to the used process parameters in a medium-sized DMD machine.

Increasing the laser speed reduces the temperature on the surface (Figure 8a,b). Subsequently, and despite the lower temperature on the surface, the cooling rate increases due to the faster movement of the heat source (Figure 8c,d). The lower the scanning speed, the larger the melt pool (Figure 8e). This will have an impact on the solidification and hence the microstructure [8,9]. 


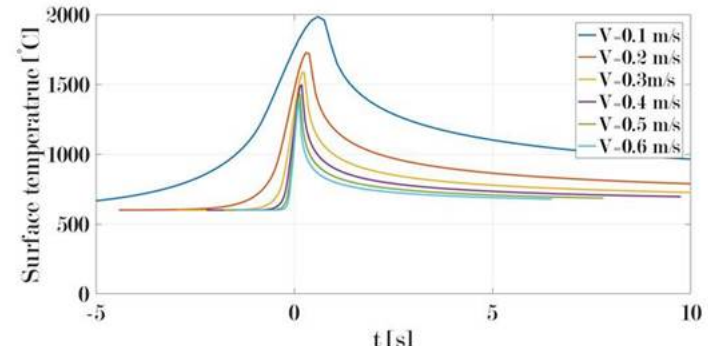

(a)

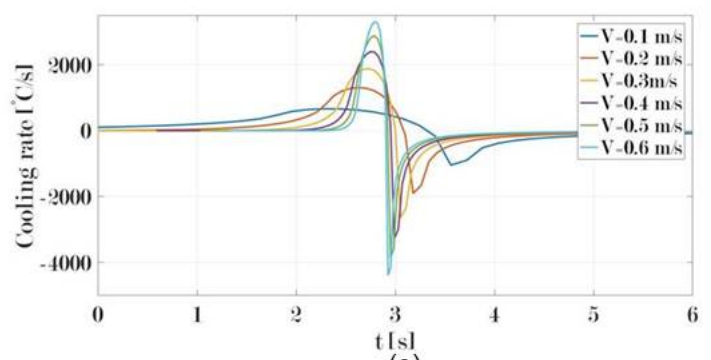

(c)

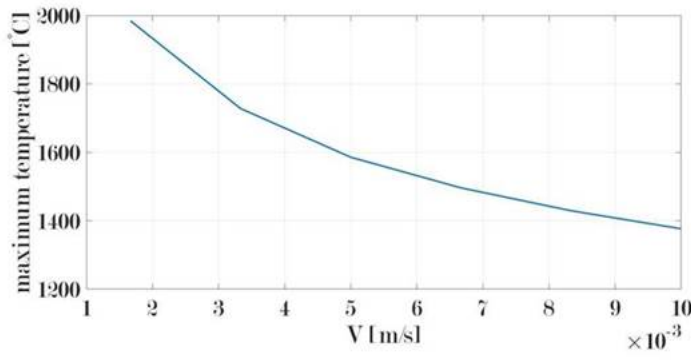

(b)

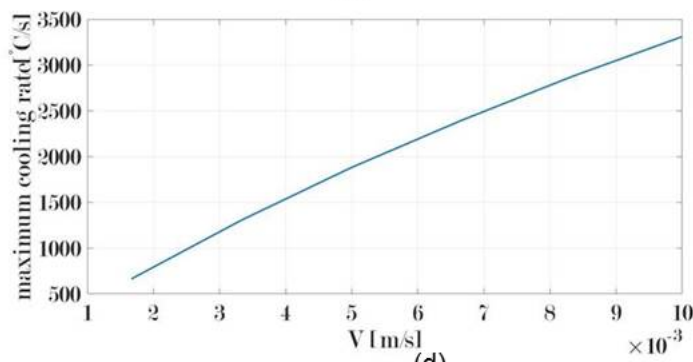

(d)

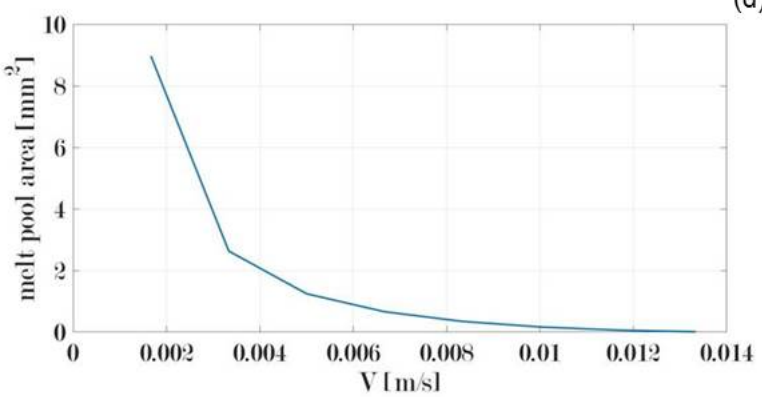

(e)

Figure 8. Simulation of the influence of the laser scanning speed on the temperature and on the melt pool cross section $(P=400 \mathrm{~W}$ ) (a) surface temperature for different scanning speeds; (b) evolution of the maximum surface temperature with the scanning speed; (c) surface cooling rate for different scanning speeds; (d) evolution of the maximum surface cooling rate with the scanning speed; and (e) evolution of the melt pool cross section with the scanning speed.

\section{Conclusions}

In this work, a numerical and an analytical model to predict the temperature during the DMD additive process are introduced. The study emphasizes the temperature gradient, the cooling rate and the melt pool size. The analytical model is based on the moving heat source assumption while the numerical model is built including the multi-physics of the laser processing. This included the phase transformation. The results of the two simulations are compared with an experimental work. Both approaches are validated and give acceptable results. The analytical model appears to be good enough to predict the critical cooling rate, which is the key factor for an online process control. Before it can be used, it still needs to be extended in a 3D geometry.

The numerical model gives information on the workpiece, especially on the melt pool and on the temperature distribution, but with a long computation time. An analytical model gives valuable information on the temperature with good precision in a short computation time. The laser power and scanning speed's influence on the temperature and cooling rate are investigated and the correlations are established. 
Acknowledgments: The authors would like to acknowledge the support from the research centre 'SFI Manufacturing' and the Material Knowledge for Robust Additive Manufacturing 'MKRAM' project (Grant No. 248243), which is sponsored by the Research Council of Norway and industrial partners. The authors from BTU would like to acknowledge funding from the EUROPEAN UNION under the European Regional Development Fund and the Federal State of Brandenburg. The authors are grateful for this financial support.

Author Contributions: The first author developed the numerical model and the second author developed the analytical model. The third author contributed to the design and the computational aspect. The fourth and fifth authors contributed to the design, writing, and editing of the manuscript.

Conflicts of Interest: The authors declare no conflicts of interest.

\section{References}

1. Leino, M.; Pekkarinen, J.; Soukka, R. The Role of Laser Additive Manufacturing Methods of Metals in Repair, Refurbishment and Remanufacturing-Enabling Circular Economy. Phys. Procedia 2016, 83, 752-760.

2. Liu, R.; Wang, Z.; Sparks, T.; Liou, F.; Newkirk, J. 13-Aerospace applications of laser additive manufacturing. In Laser Additive Manufacturing; Brandt, M., Ed.; Woodhead Publishing Series in Electronic and Optical Materials; Woodhead Publishing: Sawston, UK, 2017; pp. 351-371.

3. Bikas, H.; Stavropoulos, P.; Chryssolouris, G. Additive manufacturing methods and modelling approaches: A critical review. Int. J. Adv. Manuf. Technol. 2016, 83, 389-405.

4. Thompson, S.M.; Bian, L.; Shamsaei, N.; Yadollahi, A. An overview of Direct Laser Deposition for additive manufacturing; Part I: Transport phenomena, modeling and diagnostics. Addit. Manuf. 2015, 8, 36-62.

5. Rai, A.; Markl, M.; Körner, C. A coupled Cellular Automaton-Lattice Boltzmann model for grain structure simulation during additive manufacturing. Comput. Mater. Sci. 2016, 124, 37-48.

6. Kelly, S.M. Characterization and Thermal Modeling of Laser Formed Ti-6Al-4V. Ph.D. Thesis, Virginia Tech, Blacksburg, VA, USA, 2002.

7. Fergani, O.; Berto, F.; Welo, T.; Liang, S.Y. Analytical modelling of residual stress in additive manufacturing. Fatigue Fract. Eng. Mater. Struct. 2017, 40, 971-978.

8. Bontha, S.; Klingbeil, N.W.; Kobryn, P.A.; Fraser, H.L. Thermal process maps for predicting solidification microstructure in laser fabrication of thin-wall structures. J. Mater. Process. Technol. 2006, 178, 135-142.

9. Klingbeil, N.; Bontha, S.; Brown, C.; Gaddam, D. Effects of Process Variables and Size Scale on Solidification Microstructure in Laser-Based Solid Freeform Fabrication of Ti-6Al-4V. In Proceedings of the Solid Freeform Fabrication SFF, Austin, TX, USA, 2-4 August 2004.

10. Hoadley, A.F.A.; Rappaz, M. A thermal model of laser cladding by powder injection. Metall. Trans. B 1992, 23, 631-642.

11. Picasso, M.; Hoadley, A. Finite element simulation of laser surface treatments including convection in the melt pool. Int. J. Numer. Methods Heat Fluid Flow 1994, 4, 61-83.

12. Toyserkani, E.; Khajepour, A.; Corbin, S. 3-D finite element modeling of laser cladding by powder injection: Effects of laser pulse shaping on the process. Opt. Lasers Eng. 2004, 41, 849-867.

13. Shamsaei, N.; Yadollahi, A.; Bian, L.; Thompson, S.M. An overview of Direct Laser Deposition for additive manufacturing; Part II: Mechanical behavior, process parameter optimization and control. Addit. Manuf. 2015, 8, 12-35.

14. Cao, X.; Ayalew, B. Control-oriented MIMO modeling of laser-aided powder deposition processes. In Proceedings of the 2015 American Control Conference (ACC), Chicago, IL, USA, 1-3 July 2015; pp. 3637-3642.

15. Peyre, P.; Aubry, P.; Fabbro, R.; Neveu, R.; Longuet, A. Analytical and numerical modelling of the direct metal deposition laser process. J. Phys. D Appl. Phys. 2008, 41, 025403.

16. Elsen, M.V.; Baelmans, M.; Mercelis, P.; Kruth, J.P. Solutions for modelling moving heat sources in a semi-infinite medium and applications to laser material processing. Int. J. Heat Mass Transf. 2007, $50,4872-4882$.

17. Paul, S.; Singh, R.; Yan, W. Thermal model for additive restoration of mold steels using crucible steel. J. Manuf. Process. 2016, 24 Pt 2, 346-354.

18. Li, J.; Li, L.; Stott, F. Comparison of volumetric and surface heating sources in the modeling of laser melting of ceramic materials. Int. J. Heat Mass Transf. 2004, 47, 1159-1174. 
19. Parekh, R.; Buddu, R.K.; Patel, R. Multiphysics Simulation of Laser Cladding Process to Study the Effect of Process Parameters on Clad Geometry. Procedia Technol. 2016, 23, 529-536.

20. Vásquez, F.; Ramos-Grez, J.A.; Walczak, M. Multiphysics simulation of laser-Material interaction during laser powder depositon. Int. J. Adv. Manuf. Technol. 2012, 59, 1037-1045.

21. Kumar, A.; Roy, S. Effect of three-dimensional melt pool convection on process characteristics during laser cladding. Comput. Mater. Sci. 2009, 46, 495-506.

22. Morville, S.; Carin, M.; Peyre, P.; Gharbi, M.; Carron, D.; Le Masson, P.; Fabbro, R. 2D longitudinal modeling of heat transfer and fluid flow during multilayered. J. Laser Appl. 2012, 40, 1-9.

23. Morville, S. Modélisation Multiphysique du Procédé de Fabrication Rapide par Projection Laser en vue D’améliorer L'état de Surface Final. Ph.D. Thesis, Université de Bretagne Sud, Bretagne, France, 2012.

24. Klindworth, D.; Grepl, M.A.; Vossen, G. Certified reduced basis methods for parametrized parabolic partial differential equations with non-affine source terms. Comput. Methods Appl. Mech. Eng. 2012, 209-212, 144-155.

25. De Oliveira, U.; Ocelík, V.; Hosson, J.D. Analysis of coaxial laser cladding processing conditions. Surf. Coat. Technol. 2005, 197, 127-136.

26. Li, Y.; Zhou, K.; Tor, S.B.; Chua, C.K.; Leong, K.F. Heat transfer and phase transition in the selective laser melting process. Int. J. Heat Mass Transf. 2017, 108 Pt B, 2408-2416.

27. Carslaw, H.S.; Jaeger, J.C. Conduction of Heat in Solids; Oxford University Press: Oxford, UK, 1959.

28. Schneider, M. Laser Cladding with Powder, Effect of Some Machining Parameters. Ph.D. Thesis, University of Twente, Enschede, The Netherlands, 1998.

29. Lakso, P.; Riipinen, T.; Laukkanen, A.; Andersson, T.; Jokinen, A.; Revuelta, A.; Ruusuvuori, K. Optimization and Simulation of SLM Process for High Density H13 Tool Steel Parts. Phys. Procedia 2016, 83, 26-35.

30. Dai, K.; Shaw, L. Finite element analysis of the effect of volume shrinkage during laser densification. Acta Mater. 2005, 53, 4743-4754.

31. Pouzet, S. Fabrication Additive de Composites à Matrice Titane par Fusion Laser de Poudre Projetée. Ph.D. Thesis, ENSAM, Paris, France, 2015.

32. Yadroitsev, I.; Yadroitsava, I. Evaluation of residual stress in stainless steel 316L and Ti6Al4V samples produced by selective laser melting. Virtual Phys. Prototyp. 2015, 10, 67-76.

(C) 2017 by the authors. Licensee MDPI, Basel, Switzerland. This article is an open access article distributed under the terms and conditions of the Creative Commons Attribution (CC BY) license (http:/ / creativecommons.org/licenses/by/4.0/). 